\title{
Out of equilibrium dynamics of poly(vinyl methyl ether) segments in miscible poly(styrene)-poly(vinyl methyl ether) blends
}

\author{
Cédric Lorthioir, ${ }^{1,2}$ Angel Alegría, ${ }^{1}$ and Juan Colmenero ${ }^{1,2, *}$ \\ ${ }^{1}$ Departamento de Física de Materiales, Universidad del País Vasco (UPV/EHU) y Unidad de Física de Materiales Centro Mixto (CSIC- \\ UPV/EHU), Facultad de Química, Apartado 1072, 20080 San Sebastián, Spain \\ ${ }^{2}$ Donostia International Physics Center, Paseo Manuel de Lardizabal 4, 20018 San Sebastián, Spain \\ (Received 28 February 2003; revised manuscript received 30 May 2003; published 24 September 2003)
}

\begin{abstract}
The local dynamics of the low- $T_{g}$ component in a polymer blend, dynamically asymmetric poly(styrene)poly(vinyl methyl ether) (PS-PVME), is studied below the glass transition, via dielectric relaxation spectroscopy. A particular attention has been paid to blends with a high PS content (PS weight fraction higher than 50\%). A relaxation process, slower than the localized motions inducing the PVME secondary relaxations, is detected. Even though these blends fall out of equilibrium in this temperature regime, the structural recovery process is not efficient on the time scale of this PVME motional process. This relaxation is attributed to rather localized, weakly cooperative PVME motions resulting from the topological constraints imposed by the frozen PS chains.
\end{abstract}

DOI: 10.1103/PhysRevE.68.031805

\section{INTRODUCTION}

In miscible polymer blends with components displaying a strong difference in their glass transition temperature, a single_-but anomalously broad_-glass transition is observed by differential scanning calorimetry (DSC). However, at a molecular length scale, the mean motional rate of each component, though deeply affected by blending, remains distinct from each other. This feature has been evidenced on different asymmetric blends, such as poly(styrene)-poly(vinyl methyl ether) or poly(isoprene)-poly(vinyl ethylene), by means of dielectric relaxation spectroscopy [1], nuclear magnetic resonance (NMR) [2-5], or quasielastic neutron scattering [6], for instance. Recently, the chain connectivity has been invoked to account for this remaining difference in the segmental dynamics of both components $[7,8]$. The underlying idea is that due to the chain connectivity, the concentration of a component $A$, estimated in a small volume $\left(\sim l_{K}^{3}, l_{K}\right.$ denoting the corresponding Kuhn length) around a given segment of type $A$, is higher than the macroscopic concentration of the same polymer within the blend. The other main feature of the local dynamics in these blends is the strong broadening of the relaxation time distribution for each component, by comparison with the pure homopolymers $[1,9,10]$. This broadening continuously increases as the temperature is lowered to the glass transition. These observations are attributed to concentration fluctuations within the blends: thermodynamically driven fluctuations [11] as well as fluctuations induced by chain connectivity effects $[12,13]$. Thus, the phenomenological description of the segmental dynamics of each blend component is now well established, though a complete theoretical model is still missing.

Most of the works previously mentioned report measurements performed above or close to the glass transition $T_{g}$. By contrast, the local dynamics in polymer blends below their $T_{g}$ has been far less explored. However, this question is

\footnotetext{
*Corresponding author. Email address: wapcolej@sc.ehu.es
}

PACS number(s): 83.80.Tc, 64.70.Pf, 77.22.Gm

particularly important not only from an academic, but also industrial point of view. Indeed, polymer blends offer a good avenue to combine, at a low cost, some of the properties of the component homopolymers. For this reason, they are technologically important, but their processing and the properties of the finished products require a good knowledge of their dynamical properties, above and also below the glass transition.

One of the main difficulty is that below $T_{g}$, timedependent phenomena have also to be considered [14]. Indeed, even in the case of a single homopolymer, due to the nonequilibrium nature of the glassy state, the specific volume or the enthalpy spontaneously decreases when the system is cooled below $T_{g}$ and set under isothermal conditions. Of course, this evolution, called structural recovery process or physical aging, also induces a time dependence of the mechanical or the dielectric properties [14-17].

In the case of the blends, as the segmental mobility of the two components is different, below the calorimetric glass transition, the chain segments of the high- $T_{g}$ component could be considered as static on the time scale of the segmental motions of the low- $T_{g}$ component. In a previous paper of some of us [9], two different poly(styrene)-poly(vinyl methyl ether) (PS-PVME) blends (one with $35 \mathrm{wt} \%$ of PS, the other with $50 \mathrm{wt} \%$ ) were considered and the mean motional rate of the PS segmental motions was found to be $10^{3}-10^{4}$ times lower than the one observed on the PVME component, just at $T_{g}$. Of course, this difference is expected to be higher on increasing the content in the high- $T_{g}$ component. Besides, below $T_{g}$, the concentration fluctuations within the blends would be frozen, as evidenced by smallangle neutron scattering in the case of PS-PVME blends (one with $50 \mathrm{wt} \%$ of PS, the other with $70 \mathrm{wt} \%$ ), for instance [18]. Thus, a reasonable physical picture for the low- $T_{g}$ component dynamics in an asymmetric blend considered below its glass transition would correspond to the motional processes of mobile chains embedded within a frozen matrix formed by the high- $T_{g}$ component.

As mentioned before, the physical aging occurring below 
(a)

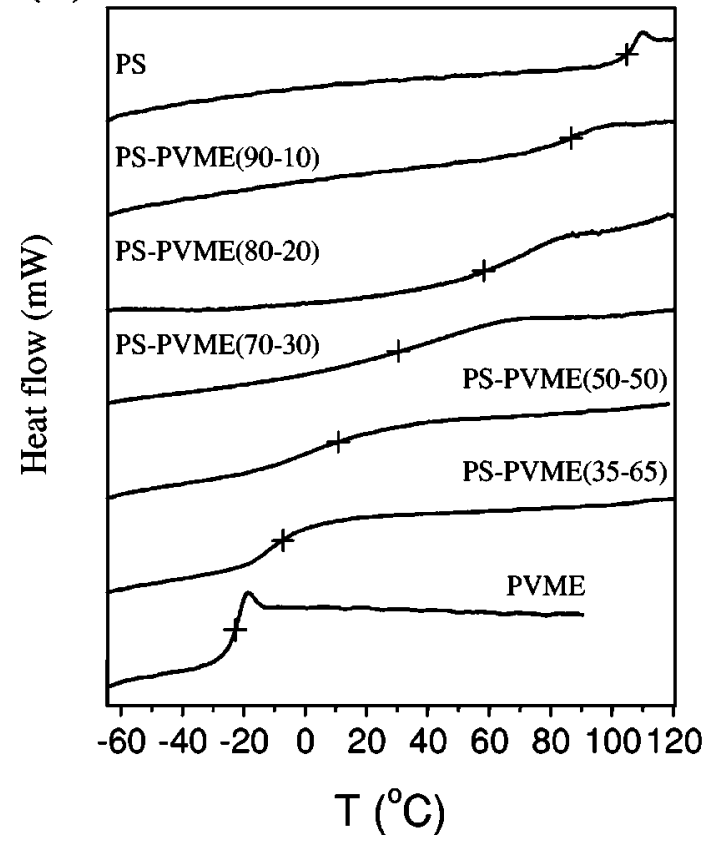

(b)

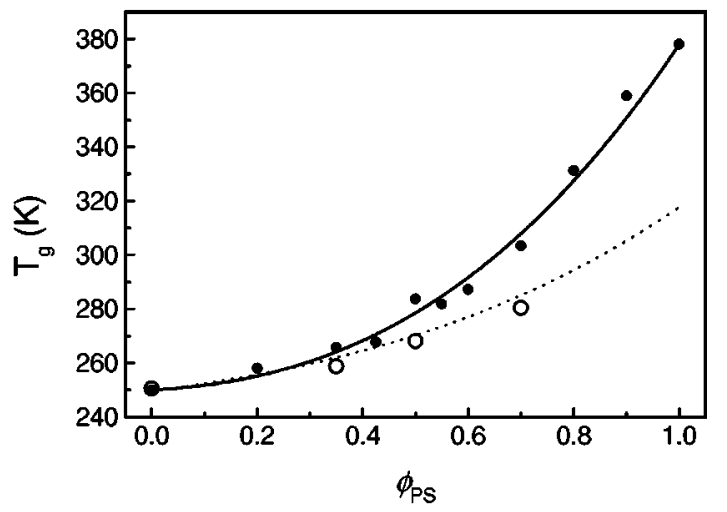

FIG. 1. (a) DSC traces for PS, PVME, and PS-PVME blends of various composition, during heating at a rate of $20 \mathrm{~K} \mathrm{~min}^{-1}$. The symbol "+" represents the midpoint of glass transition $T_{g}$; (b) composition dependence of the calorimetric glass transition temperature $T_{g}$ ( $)$, $\phi_{P S}$ denoting the PS weight fraction in the blends. The open symbols $(\bigcirc)$ stand for the dielectric glass transition temperature (at $1 \mathrm{~Hz}$ ) of PVME in the blends, deduced from the temperature of the $\alpha$-peak maximum of Fig. 3.

$T_{g}$ has to be taken into account to describe the local dynamics of the low- $T_{g}$ component. In this field, the behavior in blends rich in the low- $T_{g}$ component should differ from the one observed in blends with a large content in the high- $T_{g}$ component. Indeed, in the second case, the time scale of the structural recovery process would be essentially governed by the time scale involved in the densification of the matrix formed by the high- $T_{g}$ component. As a result, no aging effects are expected to occur on the time scale relevant for the local dynamics of the low- $T_{g}$ component. By contrast, in blends rich in the low- $T_{g}$ component or even in the corresponding homopolymer, the surrounding of the chains of the low- $T_{g}$ component will display a time evolution on the time scale of their local dynamics.

Having these ideas in mind, we explore, in this paper, the dynamical behavior of the low- $T_{g}$ component (PVME) in PS-PVME blends, below their glass transition. Indeed, this system is characterized by a strong dynamical asymmetry since both homopolymers exhibit a difference of about 123 $\mathrm{K}$ in their $T_{g}$ value. It has to be emphasized that the dynamical behavior of PVME within PS-PVME blends has been extensively investigated previously (see, for instance, Refs. $[2,3,9,10,18-20])$, but most of the works concern blends rich in PVME, studied above or close to $T_{g}$. Here, intending to limit the influence of aging processes and following the argument mentioned above, we have mainly considered PSPVME blends in the high PS concentration regime and the motional processes of PVME will be probed, below $T_{g}$, by means of broadband dielectric relaxation spectroscopy.

\section{EXPERIMENTAL SECTION}

Atactic poly(styrene) (PS, Polymer Laboratories) and poly(vinyl methyl ether) (PVME, Aldrich Chemical Co), used to prepare the blend samples, have the following molecular characteristics: for PS, weight-average molecular weight $M_{w}$ of $69800 \mathrm{~g} \mathrm{~mol}^{-1}$ and polydispersity index $I_{p}$ of 1.02; for PVME, $M_{w}=21900 \mathrm{~g} \mathrm{~mol}^{-1}$ and $I_{p}=3$. PVME, initially in aqueous solution (50 wt \%), was dried for one day at $90{ }^{\circ} \mathrm{C}$, under vacuum, to remove any traces of water. Films of PS-PVME blends with various compositions (PS weight fraction $\phi_{P S}$ ranging from $20 \%$ to $90 \%$ ) were cast from $8 \mathrm{wt} \%$ toluene solutions, since both polymers are miscible in all proportions in this solvent [21]. Casting was carried out directly on the electrodes used for dielectric relaxation measurements (diameter of $40 \mathrm{~mm}$ ), at room temperature, for about 2 days. The obtained films (thickness of about $100 \mu \mathrm{m}$ ) were then further dried in a vacuum oven. The temperature was progressively raised up to $T_{g}+30^{\circ} \mathrm{C}$ and the final temperature was maintained for 3 days. This drying process enables to remove any residual solvent within the samples and to avoid any bubble formation.

The glass transition temperature $T_{g}$ of both homopolymers and blends was determined with a Perkin-Elmer model DSC-7 instrument, at a scanning rate of $20 \mathrm{~K} \mathrm{~min}^{-1}$. These DSC measurements were performed on samples directly cut from the films investigated by dielectric relaxation spectroscopy. In Fig. 1(a) the DSC traces for PS-PVME blends are displayed with different PS weight fractions $\phi_{P S}$. In any case, a single glass transition is observed, implying, in par- 


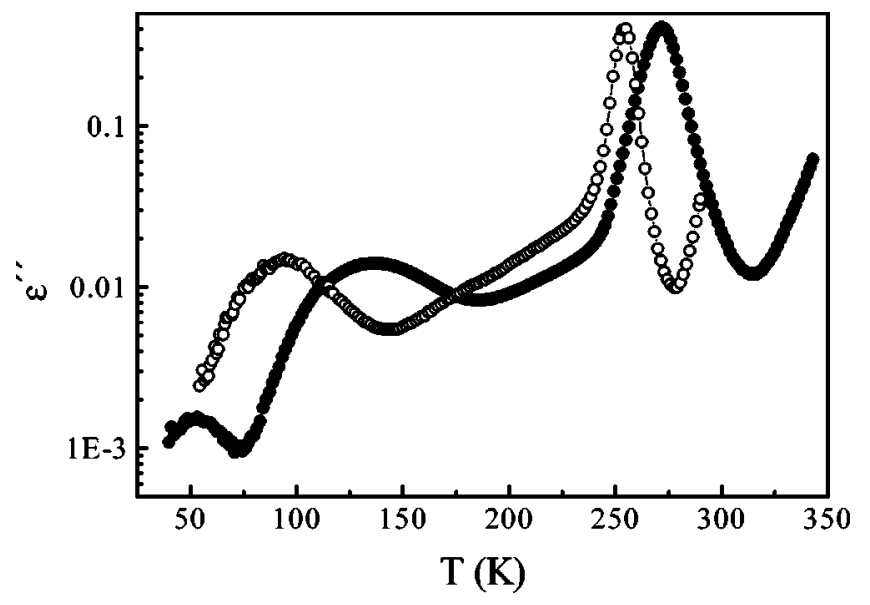

FIG. 2. Loss component $\epsilon^{\prime \prime}$ of the complex dielectric permittivity, obtained at $1 \mathrm{~Hz}(\bigcirc)$ and $1 \mathrm{kHz}(\bigcirc)$, as a function of temperature, for a PVME homopolymer.

ticular, that no phase separation occurs during the blend preparation or during the dielectric measurements. The $T_{g}$ value was determined as the temperature corresponding to half of the heat capacity change and its variation with $\phi_{P S}$ is reported in Fig. 1(b).

The complex dielectric permittivity $\epsilon^{*}=\epsilon^{\prime}-i \epsilon^{\prime \prime}$ was measured in the range $10^{-1}-10^{+7} \mathrm{~Hz}$, by using a Novocontrol high resolution dielectric analyzer (Alpha-S analyzer), for temperatures higher than $120 \mathrm{~K}$. A second electrode (diameter of $30 \mathrm{~mm}$ ) was stacked on the PS-PVME blend films and pressed under vacuum above $T_{g}$. For blends rich in PVME $\left(\phi_{P S}<50 \%\right)$, a separation of $100 \mu \mathrm{m}$ between both electrodes is maintained by means of four small Teflon spacers. The sample cell was set in a cryostat and its temperature was controlled via a nitrogen gas jet heating system coupled with a Novocontrol Quatro controller. The accuracy on the temperature value was better than $0.05 \mathrm{~K}$ on the probed temperature range $(120-363 \mathrm{~K})$. For temperatures lower than $120 \mathrm{~K}$, dielectric measurements were performed on a Solartron-Schlumberger frequency-response analyzer SI 1260 supplemented by a high-impedance preamplifier (dielectric module DM 1360, Mestec). This spectrometer is coupled to a closed-loop helium refrigeration system (CTICryogenics, Helix Technology Corporation). With this equipment, the samples were kept between two electrodes of 20 $\mathrm{mm}$ diameter, with a separation of about $100 \mu \mathrm{m}$.

It is noteworthy that in the case of PS-PVME blends, the dielectric signal mainly originates from the PVME component, due to the strong difference in the dielectric strength between PS and PVME [10].

Finally, we would like to recall the different relaxation processes that are observed in a PVME homopolymer by dielectric spectroscopy. The variation of the dielectric loss $\epsilon^{\prime \prime}$ with temperature is reported in Fig. 2 for two frequencies, 1 $\mathrm{Hz}$ and $1 \mathrm{kHz}$. The absorption peak located at $272 \mathrm{~K}$ on the curve obtained at $1 \mathrm{kHz}$ corresponds to the PVME mainchain $\alpha$ relaxation that is relevant to the glass transition. Besides, two additional secondary relaxation processes, namely, the $\beta$ and the $\gamma$ peak, are detected at $137 \mathrm{~K}$ and 53 $\mathrm{K}$, respectively. These latter are related to localized motions

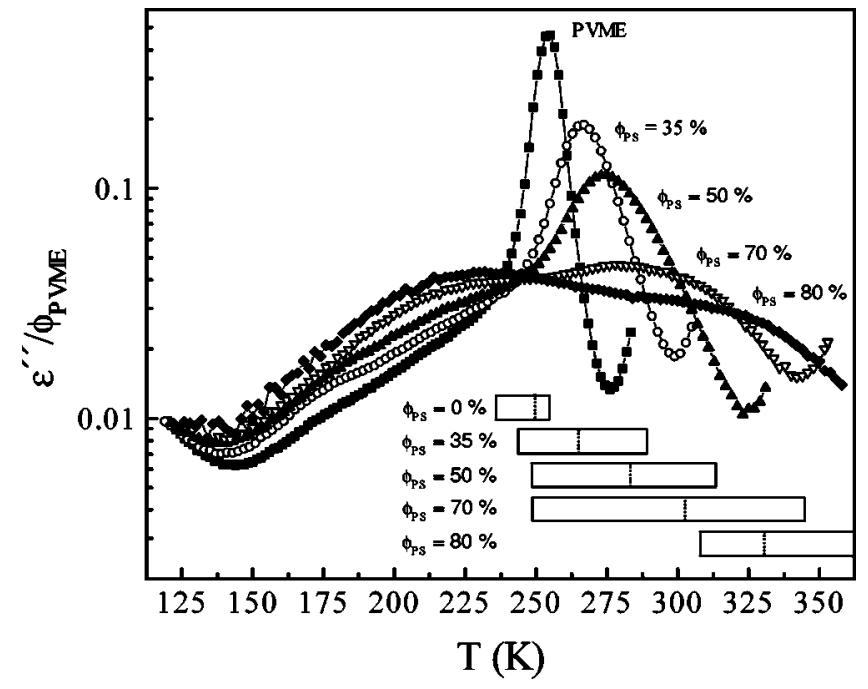

FIG. 3. Isochronal $\epsilon^{\prime \prime}(T)$ obtained at $1 \mathrm{~Hz}$ on pure PVME and PS-PVME blends with various composition $\left(\phi_{P S}=35,50,70\right.$, and $80 \mathrm{wt} \%)$. For each sample, the $\epsilon^{\prime \prime}(T)$ values have been divided by the PVME weight content $\phi_{P V M E}$. The boxes below the curves indicate the breadth of the calorimetric glass transition and the dotted lines indicate the midpoint $T_{g}$ value.

of the polar side group - $\mathrm{OCH}_{3}$. More precisely, according to a recent work [19], they could be respectively attributed to the free and restricted rotations of the methyl groups around the $\mathrm{O}-\mathrm{C}$ bonds ( $\mathrm{C}$ denoting here the PVME methine carbon). At $1 \mathrm{~Hz}$, the $\gamma$ process stands out of the probed temperature range, whereas the $\beta$ process occurs below $120 \mathrm{~K}$.

\section{RESULTS}

Figure 3 shows the temperature dependence of the dielectric loss $\epsilon^{\prime \prime}$ measured at $1 \mathrm{~Hz}$ on the PVME homopolymer as well as on PS-PVME blends of various composition $\left(\phi_{P S}\right.$ $=35 \%, 50 \%, 70 \%$, and $80 \%$ ). The frequency value was chosen low enough to allow a clear resolution of the different PVME relaxation processes. Moreover, the $\epsilon^{\prime \prime}$ values were normalized by the PVME weight fraction $\phi_{P V M E}$ to get a relevant comparison of the intensity of the relaxation peaks between the different systems. At the lowest limit of the investigated temperature range $(120 \mathrm{~K})$, the increase of $\epsilon^{\prime \prime}$ is due to the high-temperature tail of the PVME $\beta$ process (see, for comparison, Fig. 2). In this part of the spectra, the normalized $\epsilon^{\prime \prime}$ values of the different blends are clearly superposable to the one of the PVME homopolymer. This feature is confirmed by the comparison of the $\beta$ process on isochronal plots performed at higher frequencies (e.g., $10^{+5} \mathrm{~Hz}$ ) and consistent with previous studies [9]: the localized motions involved in this process are hardly affected by blending PVME with PS. By contrast, as can be seen in Fig. 3, the $\alpha$ process is strongly affected in the blends: when $\phi_{P S}$ increases, the corresponding peak starts shifting towards high temperatures and displays a strong broadening. The shift of the peak is to be related with the expected slowing down of the PVME segmental motions as PS chains are added $[2,3,9,11]$, whereas the broadening of the PVME relaxation 
time distribution can be attributed to the concentration fluctuations $[9-11,20]$. These observations are also in agreement with previous studies on other polymer blends exhibiting a large difference of $T_{g}[1,4-6]$. However, an additional process seems to grow up with the PS content. It can be unambiguously observed in the PS-PVME blend corresponding to $\phi_{P S}=50 \%$ and leads, for higher $\phi_{P S}$ values, to a doublepeak structure in the isochronal plot of Fig. 3. In this latter, the peak dominating the dielectric loss at high temperatures (above or close to $T_{g}$ ) corresponds to the $\alpha$ process described previously. In the following, we will focus on the other peak, dominating the dielectric response in the temperature range below $T_{g}$. It could be tempting to interpret the double-peak structure observed in Fig. 3 for PS-PVME blends with $\phi_{P S}>70 \%$ as a possible bimodal relaxation time distribution underlying the $\alpha$ process. Indeed, such a large and even bimodal distribution has emerged from recent models taking into account the thermodynamic fluctuation concentrations to describe the segmental dynamics in miscible blends [22]. However, these approaches assume that the considered blends are at equilibrium and therefore, are not applicable to describe the low-temperature peak since it occurs below the glass transition temperature of the blends rich in PS. Thus, to our knowledge, the low-temperature process has never been described in detail in the literature. In the following and for the sake of simplicity, we will call this process as $\alpha^{\prime}$ relaxation, but we emphasize that this notation does not mean that this relaxation is completely independent of the $\alpha$ process.

The temperature range where the $\alpha^{\prime}$-relaxation process occurs is located below the $T_{g}$ of the blends (see Fig. 3). Thus, nonequilibrium effects have to be considered, and due to the structural recovery process the dielectric loss $\epsilon^{\prime \prime}$ is expected to be time dependent [15-17]. Then, a relevant question to be addressed is the time scale involved in these changes toward the equilibrium state. For this purpose, the PVME homopolymer and different blends were annealed at a fixed temperature, below their $T_{g}$ (i.e., $T_{g}-15 \mathrm{~K}$ in any case) during $40 \mathrm{~h}$, and then $\epsilon^{\prime \prime}(T)$ was measured. Before this annealing, the samples were heated at $T_{g}+30 \mathrm{~K}$, to remove any memory effect. The temperature dependence at $1 \mathrm{~Hz}$, measured after the annealing, is reported in Fig. 4 for pure PVME and a PS-PVME blend rich in PS $\left(\phi_{P S}=80 \%\right)$. For comparison, the $\epsilon^{\prime \prime}(T)$ curves recorded at the same frequency, just before the annealing, are also plotted. In PVME, above $T_{g}, \epsilon^{\prime \prime}$ is of course the same in both unannealed and annealed states. Below $T_{g}$, a significant decrease of the signal over the whole temperature range is observed. By contrast, in the blend, the physical aging only affects the intermediate temperature range $(250-300 \mathrm{~K})$ between both relaxation peaks, which appear better resolved after annealing. The $\epsilon^{\prime \prime}$ values below $225 \mathrm{~K}$ are unchanged, which indicates that even though the $\alpha^{\prime}$ relaxation process occurs at temperatures where the blends have fallen out of equilibrium, the time scale involved in the time evolution of this process is far larger than in pure PVME.

To get a deeper understanding of the origin of the lowtemperature contribution to $\epsilon^{\prime \prime}(T)$ observed at a single fixed frequency $(1 \mathrm{~Hz})$, we have proceeded to isothermal $\epsilon^{\prime \prime}(\omega)$

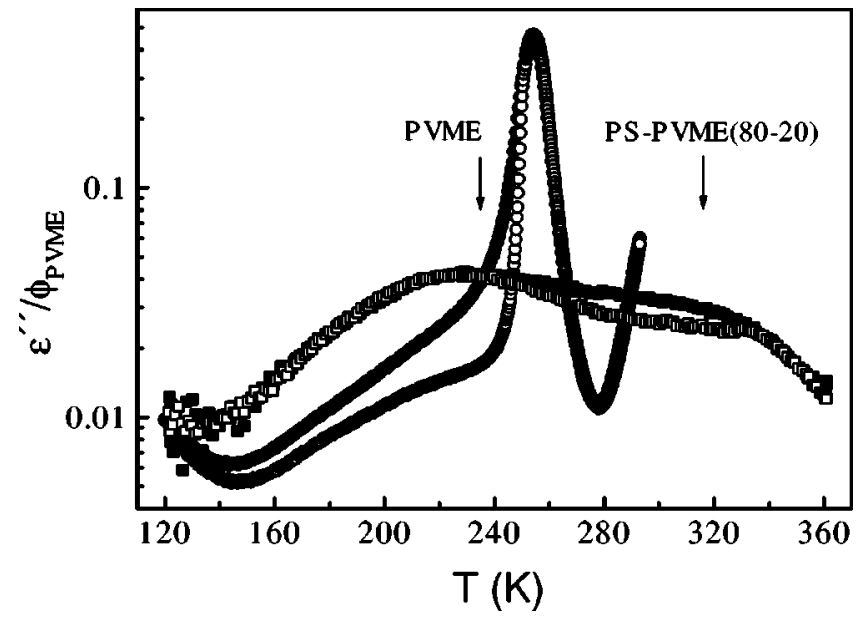

FIG. 4. Effect of physical aging on the temperature dependence of the dielectric loss $\epsilon^{\prime \prime}$ measured at $1 \mathrm{~Hz}$ : solid (open) symbols represent the data obtained on the unannealed (annealed) samples; circles correspond to PVME, and squares to the PS-PVME(80-20) blend. The arrows indicate the annealing temperatures. Details on the annealing are described in the text.

measurements, mainly focusing on PS-PVME blends with a high PS amount. The $\epsilon^{\prime \prime}(\omega)$ spectra obtained for different temperatures (between $228 \mathrm{~K}$ and $328 \mathrm{~K}$ ) on the PSPVME(80-20) blend are plotted in Fig. 5. In the whole temperature regime investigated $(223-348 \mathrm{~K})$, a single relaxation peak is detected in the spectral window $10^{-1}-10^{+7} \mathrm{~Hz}$. At $223 \mathrm{~K}$, the frequency of the peak maximum is close to $2 \mathrm{~Hz}$ : this isothermal spectrum $\epsilon^{\prime \prime}(\omega)$ can be roughly related to the maximum of $\epsilon^{\prime \prime}(T)$, occurring at 230 $\mathrm{K}$ on the isochronal plot of Fig. 4. At this temperature, the measured $\epsilon^{\prime \prime}(\omega)$ mainly originates from the $\alpha^{\prime}$-relaxation process. For temperatures around $280 \mathrm{~K}$, the $\alpha$ peak enters the considered spectral window, as can be seen in Fig. 4. This may be the origin of the flat signal observed in the low-frequency part of the dielectric loss $\epsilon^{\prime \prime}(\omega)$ for temperatures higher than $288 \mathrm{~K}$. This assumption is consistent with

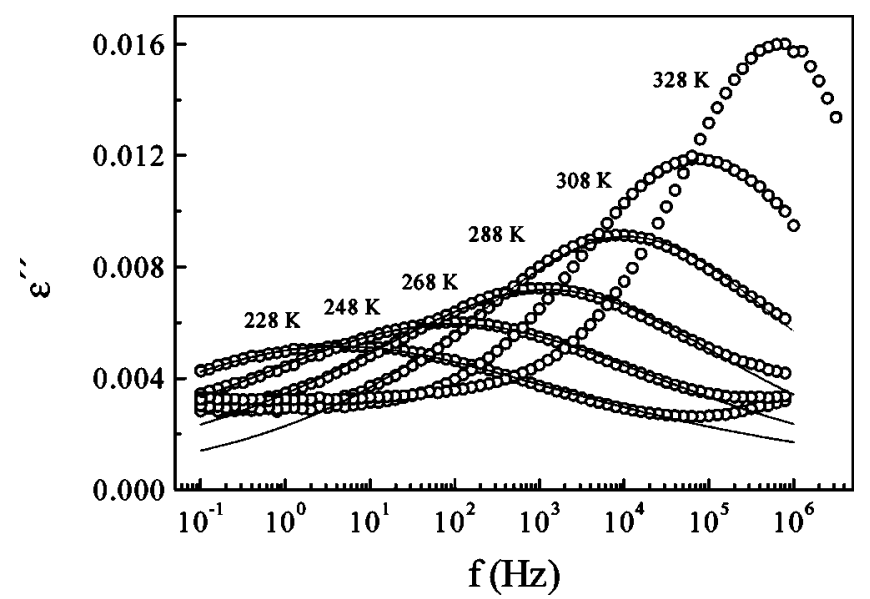

FIG. 5. Isothermal dielectric loss spectra $\epsilon^{\prime \prime}(\omega)$ recorded on a PS-PVME(80-20) blend for different temperatures. The solid lines are fits of the experimental data according to the Jonscher equation [Eq. (2)]. 


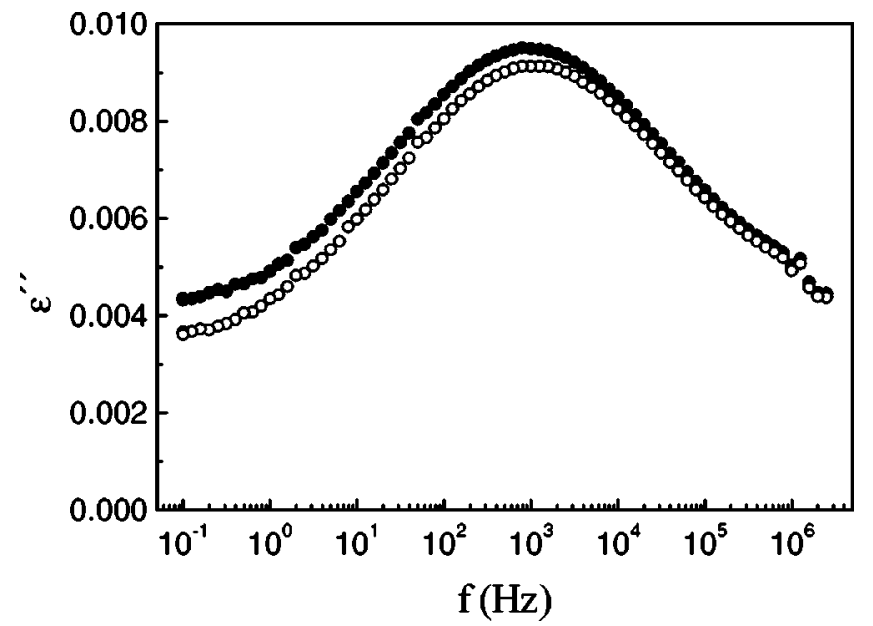

FIG. 6. Effect of physical aging on the dielectric loss spectrum $\epsilon^{\prime \prime}(\omega)$ measured at $269 \mathrm{~K}$ on the PS-PVME(80-20) blend: (a) without annealing (filled circles); (b) after an annealing time of 1 day (empty circles).

the slight increase of the level of this flat signal between 288 $\mathrm{K}$ and $348 \mathrm{~K}$. Besides, at $348 \mathrm{~K}$, a broad shoulder is observed in the low-frequency side of the $\alpha^{\prime}$ process we are interested in: it probably corresponds to the $\alpha$-peak which is indeed known to be particularly broad in the high PS concentration regime. Thus, above $288 \mathrm{~K}$, the $\epsilon^{\prime \prime}(\omega)$ spectra also include a contribution from the $\alpha$ peak and for this reason, in the following, we will restrict the probed temperature range to values lower than $288 \mathrm{~K}$.

In the following, we intend to use the isothermal measurements of $\epsilon^{\prime \prime}(\omega)$ to characterize the low-temperature dielectric response: the frequency of the peak maximum, the shape of $\epsilon^{\prime \prime}(\omega)$, and the dielectric strength $\Delta \epsilon$. Again, due to nonequilibrium effects, the question of a time-dependent characterization on the whole frequency range $10^{-1}-10^{+7} \mathrm{~Hz}$ has to be raised up. For this purpose, the PS-PVME blends with a high PS content were heated about $30 \mathrm{~K}$ above their calorimetric $T_{g}$, cooled $60 \mathrm{~K}$ below $T_{g}$, and the time variation of the dielectric loss $\epsilon^{\prime \prime}(\omega)$ was monitored for more than one day. Figure 6 reports the $\epsilon^{\prime \prime}(\omega)$ function measured on the PS-PVME(80-20) blend, before and after annealing at $T$ $=269 \mathrm{~K}$. Clearly, a small decrease of the signal below $10^{+4} \mathrm{~Hz}$ is observed, this difference increasing in the lowfrequency part. However, the peak position as well as the high-frequency part of the relaxation process are nearly unchanged. Besides, the low-frequency part of the $\epsilon^{\prime \prime}(\omega)$ peak after annealing displays, close to the peak maximum, a slope slightly higher than that before annealing. This feature, together with the decrease of the signal below $10^{+4} \mathrm{~Hz}$, suggests that the observed changes are due to the shift of a slow process out of the considered spectral window. However, the relaxation process we want to characterize is nearly unchanged. This result implies that a time-independent characterization of the low-temperature peak is allowed on a reasonable time scale. It is worth adding that during 8 months the PS-PVME(80-20) blend, let in a vacuum oven, at $20 \mathrm{~K}$ below its calorimetric $T_{g}$ does not display any additional significant changes than those presented in Fig. 6. Thus, the

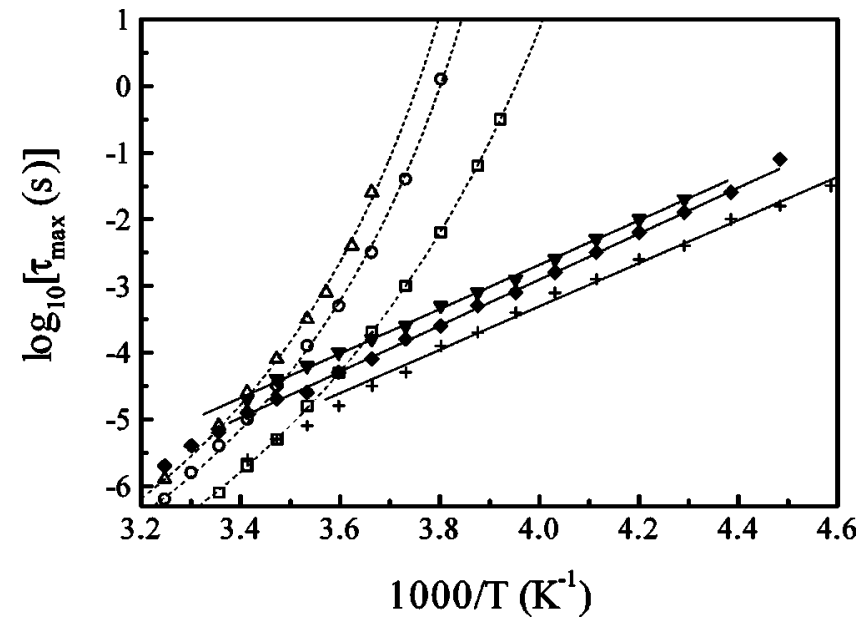

FIG. 7. Plot of the characteristic relaxation time $\tau_{\max }$, for pure PVME (empty squares) and PS-PVME blends $\left[\phi_{P S}=35\right.$ (empty circles), 50 (empty uptriangles), 70 (filled downtriangles), 80 (filled diamonds), and $90 \mathrm{wt} \%$ (cross)], as a function of temperature. The dotted lines are fits of the experimental data with a Vogel-Fulcher equation and the solid lines, with an Arrhenius law.

$\alpha^{\prime}$ process does not evolve significantly on the time scale of the isothermal measurements (each measurement requiring about $10 \mathrm{~min}$ ), allowing a characterization by timeindependent parameters.

We define the characteristic relaxation time $\tau_{\max }$ from the frequency $f_{\max }$ at the maximum of the peak observed on the isothermal measurements of $\epsilon^{\prime \prime}(\omega)\left[\tau_{\max }=1 /\left(2 \pi f_{\max }\right)\right]$. The Arrhenius plot of $\tau_{\max }$ for PS-PVME blends with $\phi_{P S}$ $=70 \%, 80 \%$, and $90 \%$ is shown in Fig. 7. For comparison, the data obtained following the same procedure on the PVME homopolymer and PS-PVME blends with a low PS content $\left(\phi_{P S}=35 \%\right.$ and $\left.50 \%\right)$ are also included. For these latter, it has to be noted that below $T_{g}$, no peak maximum that could be related to the $\alpha^{\prime}$ process was detected in the frequency range $10^{-1}-10^{+7} \mathrm{~Hz}$. For all the blends rich in PS, an Arrhenius-like behavior is observed for temperatures lower than $288 \mathrm{~K}$ :

$$
\tau_{\max }=\tau_{0} \exp \left(\frac{E_{a}}{R T}\right)
$$

The activation energy $E_{a}$ is very similar for all the blends, about $64 \mathrm{~kJ} \mathrm{~mol}^{-1}$. An independent fit of each dataset gives the values of $\tau_{0}$ and $E_{a}$ reported in Table I. It is also worthy of remark that in Fig. 7 the characteristic relaxation time of the PVME motions related to the observed process decreases with raising up the PS content.

TABLE I. Arrhenius fit parameters for the $\alpha^{\prime}$ process in PSPVME blends with PS weight fractions of $70 \%, 80 \%$, and $90 \%$.

\begin{tabular}{ccc}
\hline \hline Sample & $\tau_{0}(\mathrm{~s})$ & $E_{a}\left(\mathrm{~kJ} \mathrm{~mol}^{-1}\right)$ \\
\hline PS-PVME(70-30) & $1.07 \times 10^{-16}$ & 63.6 \\
PS-PVME(80-20) & $2.02 \times 10^{-17}$ & 66.0 \\
PS-PVME(90-10) & $5.31 \times 10^{-17}$ & 62.0 \\
\hline \hline
\end{tabular}




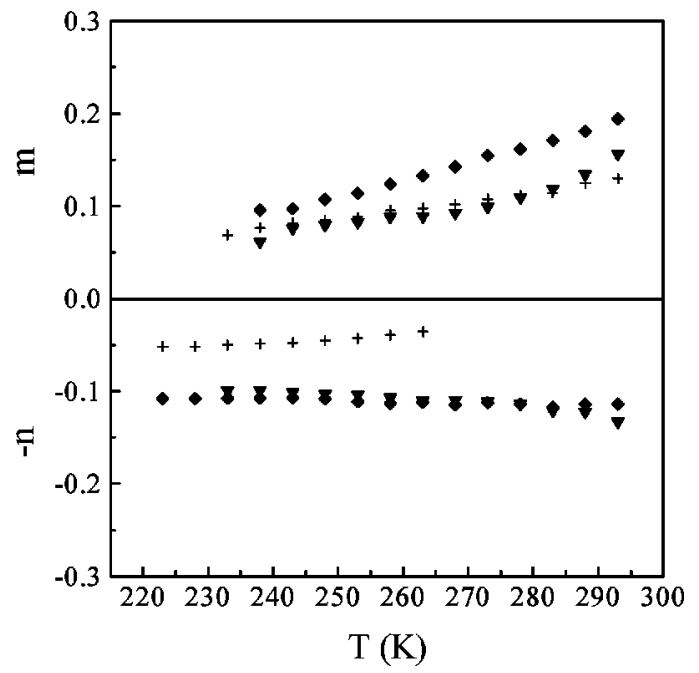

FIG. 8. Temperature dependence of shape parameters $m$ and $n$ (see definition in the text) for PS-PVME blends rich in PS $\left[\phi_{P S}\right.$ $=70$ (filled downtriangles), 80 (filled diamonds), and 90 wt $\%$ (crosses)].

The dielectric loss $\epsilon^{\prime \prime}(\omega)$ recorded on the blends can be described, around the peak maximum, by means of a Jonscher equation [23] (see solid lines in Fig. 5):

$$
\epsilon^{\prime \prime}(\omega)=\frac{2 \epsilon_{p}^{\prime \prime}}{\left(\frac{\omega}{\omega_{p}}\right)^{-m}+\left(\frac{\omega}{\omega_{p}}\right)^{n}},
$$

where the angular frequency $\omega_{p}$ is related to the loss peak maximum $\omega_{\max }$ [24], $m$ and $n$ are exponents falling in the range $0-1 ; \epsilon_{p}^{\prime \prime}$, is a constant proportional to the peak amplitude. Below (above) $\omega_{\max }$, the Jonscher equation may be approximated by two power laws, one for the low-frequency side, the other for the high-frequency one [23,25]:

$$
\begin{gathered}
\epsilon^{\prime \prime}(\omega) \propto \omega^{m} \quad\left(\omega \ll \omega_{\max }\right), \\
\epsilon^{\prime \prime}(\omega) \propto \omega^{-n} \quad\left(\omega \gg \omega_{\max }\right) .
\end{gathered}
$$

The two independent exponents $m$ and $n$ enable to probe the breadth and the asymmetry of the considered PVME relaxation process, and thus, the shape of the underlying relaxation time distribution. The temperature dependence of these shape parameters is presented in Fig. 8 for blends with $\phi_{P S}$ $=70 \%, 80 \%$, and $90 \%$. The reported values were obtained by limiting the frequency range used for the fitting procedure to $\epsilon^{\prime \prime}$ values that can be satisfactorily described by the power laws of Eq. (3). Close to $223 \mathrm{~K}(288 \mathrm{~K})$, the values of $m(n)$ were not reported since the low- (high-) frequency tail of $\epsilon^{\prime \prime}(\omega)$ comes out of the probed spectral window, leading to unprecise shape exponent values. In addition, as for the PSPVME(90-10) blend, a greater incertitude affects both shape exponent values, due to the weakness of the dielectric signal. Clearly, the shape of $\epsilon^{\prime \prime}(\omega)$ does not depend much on temperature (between $223 \mathrm{~K}$ and $288 \mathrm{~K}$ ) and is nearly unaffected

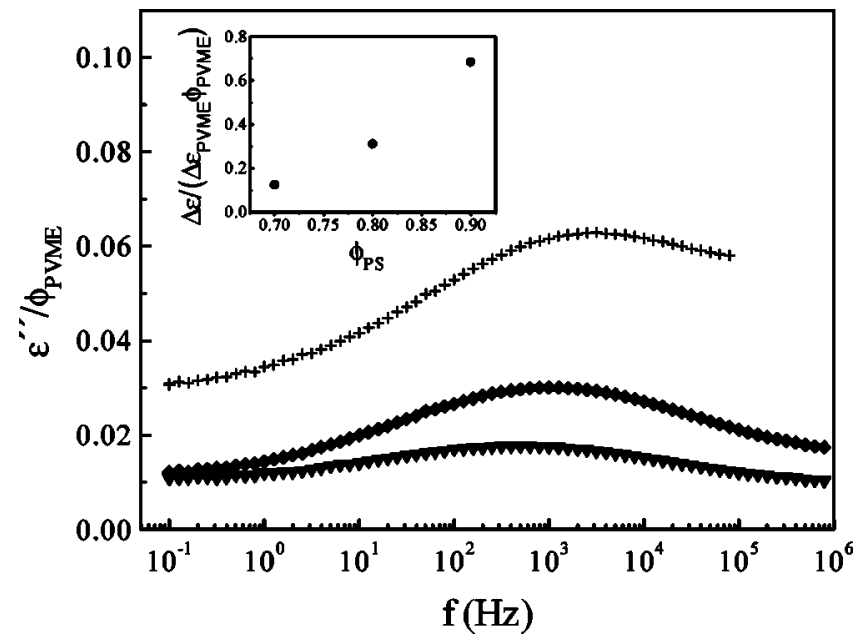

FIG. 9. Dielectric loss spectra $\epsilon^{\prime \prime}(\omega)$, normalized by the PVME weight fraction $\phi_{P V M E}$, recorded at $268 \mathrm{~K}$, on PS-PVME blends with $\phi_{P S}=70 \%$ (filled downtriangles), $80 \%$ (filled diamonds), and 90\% (crosses). Inset: Variation with the blend composition of the relative dielectric strength, i.e., the dielectric strength $\Delta \epsilon$ of the $\alpha^{\prime}$ process over the dielectric strength $\Delta \epsilon_{P V M E} \phi_{P V M E}$ of the total number of PVME segments within the blend.

by the amount of PS. Besides, the relaxation peak is very broad $(m \simeq n \simeq 0.1)$ and rather symmetric $(m \simeq n)$.

Finally, another quantity of interest is the dielectric strength $\Delta \epsilon$ related to this relaxation peak. $\Delta \epsilon$ was estimated by using the equation

$$
\Delta \epsilon=\frac{2}{\pi} \int \epsilon^{\prime \prime}(\omega) d(\ln \omega) .
$$

In Eq. (4), the experimental $\epsilon^{\prime \prime}(\omega)$ are replaced by the analytical expression deduced from the fit by a Jonscher function. At $268 \mathrm{~K}$, for PS-PVME blends with $\phi_{P S}=70 \%, 80 \%$, and $90 \%$, the dielectric loss peak $\epsilon^{\prime \prime}(\omega)$ is centered in the considered spectral window $10^{-1}-10^{+7} \mathrm{~Hz}$ (see Fig. 9), thus leading to a more precise determination of $\Delta \epsilon: 0.030$ for $\phi_{P S}=70 \%, 0.050$ for $\phi_{P S}=80 \%$, and 0.056 for $\phi_{P S}$ $=90 \%$. In the case of the PS-PVME(90-10), due to the weakness of the dielectric signal, the experimental data used for the evaluation of $\Delta \epsilon$ were limited, in the high-frequency side, to $10^{+5} \mathrm{~Hz}$. The $\Delta \epsilon$ values of the $\alpha^{\prime}$ process were compared to the dielectric strength related to the total number of PVME segments in the blends. This latter is estimated as the product of the dielectric strength of pure PVME $\left(\Delta \epsilon_{P V M E}=0.817\right.$ [9]) by the PVME weight fraction. The increase of the corresponding relative dielectric strength, also evidenced in the inset of Fig. 9, shows that the amount of PVME chain segments contributing to the $\alpha^{\prime}$-relaxation peak is more and more important as the PS content is raised up.

\section{DISCUSSION}

Our dielectric measurements have evidenced that in addition to the $\beta$ and $\gamma$ peaks, another relaxation process occurs, below $T_{g}$, in the blends rich in PS. The corresponding di- 
electric loss $\epsilon^{\prime \prime}(\omega)$ is broad, symmetric, and follows an Arrhenius-like temperature dependence. These three features indicate that this relaxation process displays similarities with the characteristics of a secondary relaxation, and thus, the underlying motional process should be rather localized. However, it has to be clearly distinguished from the $\beta$ peak observed on PVME and on all PS-PVME blends. Indeed, this latter occurs below $120 \mathrm{~K}$ at $1 \mathrm{~Hz}$. Besides, its activation energy $E_{a}$ is of $21.7 \mathrm{~kJ} \mathrm{~mol}^{-1}$ [9], i.e., about three times lower than that of the considered relaxation process. In particular, this difference between the $E_{a}$ values of both processes suggests that the PVME motions involved in the $\alpha^{\prime}$ relaxation extend on a larger length scale than the motions related to the PVME $\beta$ process (fluctuations of the pendant group $\left.-\mathrm{OCH}_{3}[19]\right)$. In addition, it is noteworthy that the $\beta$ and $\gamma$ peaks of PVME in PS-PVME blends are not dependent on the PS concentration, in contrast with the $\alpha^{\prime}$ peak.

In the PS-PVME blends with $\phi_{P S}$ higher than $70 \%$, the PVME chain segments are, on average, surrounded by PS chains or subchains. Besides, in this composition range, the effective glass transition of PS is estimated, through the model proposed by Lodge and McLeish [7], to stand above $325 \mathrm{~K}$. (More precisely, it is expected to increase from $325 \mathrm{~K}$ to $355 \mathrm{~K}$ when $\phi_{P S}$ is varied from $70 \%$ to $90 \%$ ) [8]. Thus, in the probed temperature range ( $T$ lower than about $280 \mathrm{~K}$ ), the PVME segmental motions giving rise to the $\alpha^{\prime}$-relaxation process occur in an environment consisting of "frozen" PS chains. As a result, cooperative motions involving both PS and PVME chain portions are not possible. The only PVME main-chain motions that would be possible are conformational transitions (or rotational fluctuations) implying very few monomer units and compatible with the topological constraints induced by the PS chains. Thus, by constraining the PVME segmental motions, the PS chains would promote local rearrangements, which are consistent with the behavior of the low-temperature peak previously described (similarities with a secondary process).

Now, we will focus on the temperature dependence observed in the PS-PVME blends rich in PS, below $T_{g}$. Let us first come back to the case of a homopolymer. Above the glass transition $T_{g}$, the temperature dependence of the $\alpha$-relaxation time $\tau$ of polymers can be described by a Vogel-Fulcher law:

$$
\tau(T)=\tau_{0} \exp \left(\frac{B}{T-T_{0}}\right)
$$

Such a dependence can be rationalized by using the Adam and Gibbs theory [26], based on the configurational entropy $S_{c}$ of the system. In this approach, the reorientational time of a given unit is related to $S_{c}$ according to

$$
\tau(T)=\tau_{0} \exp \left(\frac{N_{A} s_{c}^{*} \Delta \mu}{k_{B} T S_{c}}\right)
$$

where $s_{c}^{*}$ is the entropy of the minimum number of particles involved in the cooperative motions; $\Delta \mu$, the molar elementary activation energy; $\tau_{0}$, the reciprocal of a frequency attempt; $N_{A}$, Avogadro's number, and $k_{B}$, Boltzmann's con- stant. It has to be emphasized that when the Adam and Gibbs equation is applied far below $T_{g}$, where the configurational entropy is expected to be temperature independent, an Arrhenius law results $[15,27,28]$. According to Eq. (6), the corresponding activation energy of this Arrhenius law would be expressed as:

$$
E_{a}^{A G}=\frac{N_{A} s_{c}^{*} \Delta \mu}{S_{c}^{\text {frozen }}} .
$$

It should be noted that the PVME segmental motions far below $T_{g}$ in the homopolymer are extremely slow $[15,16]$. This situation can be related to that occurring in PS-PVME blends rich in PS, far below $T_{g}$, where the time scale of the PVME segmental motions is rather low. Following our interpretation, in that temperature range, the PVME motions take place in a matrix made of frozen PS chains. Thus, the configurational entropy in that temperature range would be essentially temperature independent. Taking this into account, the activation energy $E_{a}$ of the $\alpha^{\prime}$ process should be of the same order of magnitude as $E_{a}^{A G}$. By using the parameters corresponding to the PVME homopolymer [15], the resulting value of $E_{a}^{A G}$ is about $57 \mathrm{~kJ} \mathrm{~mol}^{-1}$ which, indeed, is not very different from the $E_{a}$ value deduced from the temperature dependence of $\tau_{\max }$ in the blends ( $E_{a}$ was found to be of about $64 \mathrm{~kJ} \mathrm{~mol}^{-1}$ ). This difference can be rationalized by considering that the value of $S_{c}^{\text {frozen }}$ for PVME could be slightly different from those corresponding to the blends. This interpretation is also consistent with the fact that the apparent activation energy in the blends has been found to be nearly independent of composition.

Until now, for the sake of simplicity, we have only considered the mean PS weight fraction over the whole blend and disregarded the effect of the concentration fluctuations. However, these latter should be at least partially responsible for the large breadth of the $\alpha^{\prime}$-relaxation process: depending on the local PS concentration, the corresponding PVME motions will be more or less constrained.

The previously described process can also be observed for the temperature variation of $\epsilon^{\prime \prime}$ measured at $1 \mathrm{~Hz}$ on the PS-PVME(50-50) blend (see Fig. 3), though it appears far less pronounced than for higher $\phi_{P S}$ values. Indeed, due to concentration fluctuations, some regions within the blend experience local PS weight fractions much higher than 50\%. In these regions, the PVME motions will also give rise to an additional relaxation process, as explained for blends rich in PS. This is consistent with previous studies of PS-PVME blends rich in PVME ( $\phi_{P S}=35 \%$ and 50\%) [9], which have suggested that some of the PVME segments within these mixtures display motional processes faster than those in pure PVME. Besides, in the PS-PVME(50-50) blend, the $\alpha^{\prime}$ peak can be observed on isothermal $\epsilon^{\prime \prime}(\omega)$ measurements, far below the glass transition temperature, at the border of our accessible frequency range. Assuming a peak shape independent of temperature in that range, a frequency-temperature superposition of the high-frequency tail of this peak leads to an activation energy of about $51 \mathrm{~kJ} \mathrm{~mol}^{-1}$, consistent with the value obtained for blends rich in PS. 
The interpretation of the molecular motions inducing the $\alpha^{\prime}$-relaxation process is also consistent with the results obtained with our physical aging experiments. Indeed, in PSPVME blends rich in PS, the evolution toward the equilibrium state during annealing is driven by the structural recovery process of the PS chains. In our experiments, the annealing temperature was at least $15 \mathrm{~K}$ lower than the calorimetric $T_{g}$, and thus much lower than the PS effective glass transition temperature. Thus, the time scale involved by the aging of the PS matrix is far higher than the considered annealing time, resulting in the absence of any significant effect of annealing on the shape of the observed low-temperature $\epsilon^{\prime \prime}(T)$ peak. This behavior has to be contrasted with the case of the PVME homopolymer or the blends rich in PVME. In these latter, each PVME chain is, on average, mainly surrounded by other PVME chains and thus, its environment will undergo a structural relaxation over the chosen annealing time (see the case of PVME in Fig. 4).

At this stage, it is interesting to compare our results with previous studies on physical aging of PS-PVME blends. In particular, Cowie and Ferguson have reported enthalpy relaxation measurements on a PS-PVME(50-50) blend which were analyzed through two models: the Cowie-Ferguson model and the Petrie-Marshall one [29]. They proved that the PVME component is mainly responsible for the physical aging process they monitor, which allows us to compare their data with our dielectric measurements, mainly sensitive to PVME. The analysis with the Petrie-Marshall model leads to an apparent activation energy $E_{H}$ for enthalpy relaxation of $929 \mathrm{~kJ} \mathrm{~mol}^{-1}$, value determined within the temperature range $250-270 \mathrm{~K} . E_{H}$ corresponds to the activation energy for enthalpy relaxation after complete physical aging of the blend. This value should be compared to the apparent activation energy deduced from our dielectric data on the PSPVME(50-50) blend, using an extrapolation of the VogelFulcher fit of $\tau_{\max }$ (see Fig. 7). The thus obtained $E_{a}$ values range between about 1160 and $350 \mathrm{~kJ} \mathrm{~mol}^{-1}$ for $T$ varying from 250 to $270 \mathrm{~K}$, which indeed is consistent with the $E_{H}$ value reported in Ref. [29]. In addition, using the CowieFerguson model, the authors fitted the enthalpic relaxation measurements using a Kolhrausch-Williams-Watts function, its characteristic time being $\tau_{c}$. By approximating the temperature dependence of $\tau_{c}$ between 250 and $270 \mathrm{~K}$ via a rough Arrhenius fit, we found an apparent activation energy for the out of equilibrium relaxation in the blend of about $65 \mathrm{~kJ} \mathrm{~mol}^{-1}$ : this value is in close agreement with the activation energy $E_{a}$ of the $\alpha^{\prime}$ process deduced following the out of equilibrium PVME segmental dynamics. It is lower than the one found in pure PVME in the glassy state $\left(125 \mathrm{~kJ} \mathrm{~mol}^{-1}\right)$ by means of time domain dielectric measurements [16].

One basic question that remains concerns the values of the relaxation times involved in this process. Indeed, a speeding up of the PVME localized motions is observed when the PS content is increased, as can be seen in Fig. 7 or Fig. 9. This suggests that confinement effects could take place within these blends. Indeed, following our previous view, PVME chains would be confined in a threedimensional solidlike PS matrix. Here, it is not obvious to define the confining length $d$ of the PVME segments. But, in a first approach, considering that each PVME chain is surrounded by PS ones, the confinement length would be of the order of $10 \AA$, i.e., twice the typical interchain distance in a homopolymer. This confining length is analogous to that considered in previously studied systems such as nanocomposites, made of polymer chains intercalated within the inner galleries of silicate layers [30]. In these nanocomposites, the confining distance is also close to $10 \AA$, but one strong difference in the case of the blends is that the confinement is very ill defined.

Confined polymer chains are known to display a structural and dynamical behavior different from the bulk state. It is well admitted now that the segmental motions can be speeded up or slowed down, due to a competition between pure (geometric) confinement and the effects of the interactions between the confined polymer chains and the confining surfaces. Having this point in mind and following the analogy with polymers confined in well-defined matrixes, one can assume that, in a first approach, no "interface" effect by the PS chains on the PVME segmental dynamics has to be taken into account. Indeed, in the case of PS-PVME blends, only weak van der Waals interactions between the PS phenyl ring and the PVME methoxyl group occur, as evidenced by two-dimensional (2D) NMR experiments [31]. In that context, it is expected that pure confinement induces a speeding up of the segmental dynamics, by comparison with the unconfined state (PVME homopolymer). This would rationalize the observed speeding up in the blends rich in PS. However, this analogy with systems where the confining distance is uniform has to be taken with great care. Indeed, even for a confined simple liquid, recent molecular dynamics simulations have evidenced that, depending on the roughness of the confining walls (smooth or rough), a speeding up or a slowing down could be observed in the relaxation dynamics of the liquid [32]. Further dielectric spectroscopy experiments are currently being developed on these PS-PVME blends to clarify the origin of the speeding up we observe.

Finally, the occurrence of PVME segmental motions, related to the $\alpha^{\prime}$ process, within the blends rich in PS could be of particular interest for the mechanical properties of these materials below their $T_{g}$. It turns out that blending appears as an "artificial" way of inducing molecular mobility in the glassy state. This feature could be of great interest for possible industrial applications of these multicomponent systems.

\section{CONCLUSION}

We have investigated the motional processes of the low$T_{g}$ component (PVME) within the homogeneous phase of miscible polymer blends, dynamically asymmetric (PSPVME), below their glass transition. Blends rich in the high$T_{g}$ component were mainly considered. In addition to the well-known PVME secondary relaxations, another relaxation process called by us $\alpha^{\prime}$ relaxation is detected in these blends. This relaxation is related to motions of the PVME chain segments constrained by the matrix formed by the frozen PS chains. With this respect, the molecular nature of this $\alpha^{\prime}$ 
relaxation has certainly to be related with the segmental $\alpha$ relaxation of PVME, but in a constrained geometry. This attribution is consistent with the fact that the structural recovery process, essentially driven by the PS matrix, is much slower than the time scale involved by these PVME motions. Besides, a speeding up of the PVME motional process by increasing the PS concentration of the blend is observed. Similarities with systems of polymer chains confined in wellordered matrixes are discussed and suggest that this speeding up would also result from the confinement of the PVME chains by the PS matrix.

\section{ACKNOWLEDGMENTS}

The authors acknowledge the University of the Basque Country (Project No. 9/UPV00206.215-13568/2001) and the Spanish Ministry of Science and Technology (Project No. MAT 2001/0070) for their support.
[1] F. Alvarez, A. Alegría, and J. Colmenero, Macromolecules 30, 597 (1997).

[2] C. LeMenestrel, A.M. Kenwright, P. Sergot, F. Lauprêtre, and L. Monnerie, Macromolecules 25, 3020 (1992).

[3] K. Takegoshi and K. Hikichi, J. Chem. Phys. 94, 3200 (1991).

[4] B. Min, X.H. Qiu, M.D. Ediger, M. Pitsikalis, and N. Hadjichristidis, Macromolecules 34, 4466 (2001).

[5] S. Saxena, D. Cizmeciyan, and J.A. Kornfield, Solid State Nucl. Magn. Reson. 12, 165 (1998).

[6] S. Hoffmann, L. Willner, D. Richter, A. Arbe, J. Colmenero, and B. Farago, Phys. Rev. Lett. 85, 772 (2000).

[7] T.P. Lodge and T.C.B. McLeish, Macromolecules 33, 5278 (2000).

[8] E. Leroy, A. Alegría, and J. Colmenero, Macromolecules 35, 5587 (2002).

[9] I. Cendoya, A. Alegría, J.M. Alberdi, J. Colmenero, H. Grimm, D. Richter, and B. Frick, Macromolecules 32, 4065 (1999).

[10] A. Zetsche, F. Kremer, W. Jung, and H. Schulze, Polymer 31, 1883 (1990).

[11] A. Zetsche and E.W. Fischer, Acta Polym. 45, 168 (1994).

[12] S. Salaniwal, R. Kant, R.H. Colby, and S.K. Kumar, Macromolecules 35, 9211 (2002).

[13] E. Leroy, A. Alegría, and J. Colmenero, Macromolecules (to be published).

[14] L.C.E. Struik, Physical Aging in Amorphous Polymers and Other Materials (Elsevier, Amsterdam, 1978).

[15] A. Alegría, L. Goitiandía, I. Tellería, and J. Colmenero, Recent Res. Dev. Macromol. Res. 3, 49 (1998).

[16] A. Alegría, E. Guerrica-Echevarría, L. Goitiandía, I. Tellería, and J. Colmenero, Macromolecules 28, 1516 (1995).
[17] A. Alegría, L. Goitiandía, I. Tellería, and J. Colmenero, Macromolecules 30, 3881 (1997).

[18] H. Takeno, S. Koizumi, H. Hasegawa, and T. Hashimoto, Macromolecules 29, 2440 (1996).

[19] O. Urakawa, Y. Fuse, H. Hori, Q. Tran-Cong, and O. Yano, Polymer 42, 765 (2001).

[20] J.A. Pathak, R.H. Colby, G. Floudas, and R. Jérôme, Macromolecules 32, 2553 (1999).

[21] M. Bank, J. Leffingwell, and C. Thies, Macromolecules 4, 43 (1971).

[22] S.K. Kumar, R.H. Colby, S.H. Anastasiadis, and G. Fytas, J. Chem. Phys. 105, 3777 (1996).

[23] A.K. Jonscher, Dielectric Relaxation in Solids (Chelsea Dielectrics Press, London, 1983).

[24] More precisely, $\omega_{\max }=\omega_{p}{ }^{m+n} \sqrt{m / n}$ and $\omega_{\max }$ reduces to $\omega_{p}$ in the case of a symmetric loss peak only.

[25] Broadband Dielectric Spectroscopy, edited by F. Kremer and A. Schönhals (Springer-Verlag, Berlin, 2003).

[26] G. Adam and J.H. Gibbs, J. Chem. Phys. 43, 139 (1965).

[27] L. Goitiandía and A. Alegría, J. Non-Cryst. Solids 287, 237 (2001).

[28] S. Matsuoka, Relaxation Phenomena in Polymers (Hanser, Munich, 1992).

[29] J.M.G. Cowie and R. Ferguson, Macromolecules 22, 2312 (1989).

[30] S.H. Anastasiadis, K. Karatasos, G. Vlachos, E. Manias, and E.P. Giannelis, Phys. Rev. Lett. 84, 915 (2000).

[31] P.A. Mirau and F.A. Bovey, Macromolecules 23, 4548 (1990).

[32] P. Scheidler, W. Kob, and K. Binder, Europhys. Lett. 59, 701 (2002). 